\title{
How task errors affect subsequent behavior: Evidence from distributional analyses of task-switching effects
}

\author{
Marco Steinhauser and Ronald HüBner \\ Universität Konstanz, Konstanz, Germany
}

\begin{abstract}
Switch costs in task switching are often assumed to reflect the strengthening of task-related associations. Recently, we provided evidence that committing an error leads to the strengthening of the wrong task (Steinhauser \& Hübner, 2006). In the present study, we report how error-induced control compensates for the effects of error strengthening. We hypothesized that, although error detection cannot prevent an error from being strengthened, it initiates processes that suppress the negative consequences of error strengthening on subsequent trials. Because error-induced control is known to operate slowly, we predicted that a compensatory effect should be observable only for slow responses. In three task-switching experiments, these predictions were confirmed by distributional analyses. The results extend our understanding of the interplay between procedural learning and executive control.
\end{abstract}

Endogenous control is a prerequisite of goal-directed performance. When control fails, behavior is no longer driven by goals, but rather by external stimuli. This can be demonstrated not only in patients with frontal brain lesions (Lhermitte, 1983; Norman \& Shallice, 1986), but also in healthy persons under time pressure or distraction (Monsell, 1996; Norman, 1981; Reason, 1990). For instance, so-called capture errors occur when stimuli evoke an associated but unintended behavior. This is nicely illustrated by the classic example, described by William James, in which an absent-minded person goes to the bedroom to get dressed for dinner. After a while, however, he finds himself lying in bed dressed for sleep (James, 1890).

The aim of the present study was to investigate the consequences of such capture errors for subsequent performance. A correct response to a stimulus usually leads to an incidental learning of the current processing episode (Logan, 1988) or to a strengthening of the applied task (Meiran, 2000). In a recent study using the task-switching paradigm, we observed that, when an error occurs because the wrong task was accidentally applied, the wrong task is strengthened, which then impairs the execution of the originally intended task on a subsequent trial (Steinhauser \& Hübner, 2006). In the present study, we addressed the question of whether some processes can counteract these negative consequences of incidental error learning.

\section{Task Switching and}

\section{Erroneous Task Strengthening}

The task-switching paradigm was introduced in order to examine the control processes involved in switching between task sets (Allport, Styles, \& Hsieh, 1994; Meiran, 1996; Rogers \& Monsell, 1995). The paradigm requires that two or more tasks be performed in alternation. If the performance on task-switch trials is compared with that on task-repetition trials, so-called switch costs are usually observed, which were initially thought to reflect top-down control involved in task-set reconfiguration (Meiran, 1996; Rogers \& Monsell, 1995; Steinhauser, Maier, \& Hübner, 2007). However, it soon became obvious that the switch costs are also related to memory processes - that is, to priming or the associative strengthening of tasks. According to some accounts (e.g., Meiran, 2000; Schuch \& Koch, 2003; Steinhauser \& Hübner, 2006), task execution implies that task rules (such as "press left key when the stimulus is an even number") of the current task are strengthened, whereas rules of competing tasks are weakened. Switch costs emerge because repeating the previously strengthened task is beneficial, whereas switching toward the previously weakened task is costly. From this perspective, switch costs can be viewed as an indicator of procedural learning at the level of tasks. ${ }^{1}$

To investigate the strengthening mechanisms in more detail, we recently analyzed the switch effects on trials following errors (Steinhauser \& Hübner, 2006). We found that after an error, the usually observed switch costs turned into switch benefits - that is, performance was improved on task-switch trials relative to task-repetition trials. These reversed effects, relative to those after correct responses, can easily be explained by the strengthening account. If an error is caused by task confusion - that is, by accidentally applying the wrong task (see Meiran \& Daichman, 2005) - the wrong task is then strengthened instead of the correct one. As a consequence, a subsequent "switch" to the erroneously applied task is now beneficial, whereas a "repetition" of the previously intended task is costly. In further experiments, we

M. Steinhauser, marco.steinhauser@uni-konstanz.de 
observed that the reversed effects even occurred when the participants were aware of the errors (as measured by error signaling; see also Steinhauser, Maier, \& Hübner, 2008). In contrast, if errors were corrected immediately after the response, the usual switch costs occurred on the subsequent trial. This implies that the strengthening of the wrong task from an error can be compensated for by an immediate correction. In contrast, merely detecting the error is not sufficient to prevent the error from being strengthened.

From these results, several important conclusions can be drawn regarding the conditions under which strengthening occurs, as well as regarding the nature of the strengthening mechanism. First, it seems that strengthening is triggered by the production of a task-relevant response. This led us to propose a response-based strengthening account, which assumes that, whenever a response is produced, the task by which this response was derived is strengthened. Producing an overt task-relevant response serves as a cue to initiate strengthening of the currently relevant task rules (for similar ideas, see Philipp, Jolicœur, Falkenstein, \& Koch, 2007; Schuch \& Koch, 2003). Second, task strengthening is incidental and unsupervised by nature (as in, e.g., unsupervised Hebbian learning). As a consequence, an erroneously applied task is strengthened in the same way as a correctly applied task. This is consistent with the suggestion that implicit memory works in an unsupervised way (see, e.g., Baddeley \& Wilson, 1994; McClelland, 2001).

\section{Is There Compensatory Control of Error Learning?}

The results described so far demonstrate a serious shortcoming of unsupervised learning: It does not prevent errors from being learned. Of course, error learning does not impair overall performance in a task-switch experiment, because the costs on task-repetition trials are counterbalanced by the benefits on task-switch trials after an error. However, it is plausible to assume that the proper function of task strengthening is skill acquisition and automatization of task performance in the course of repeated practice (Cohen, Dunbar, \& McClelland, 1990). In this situation, error learning is clearly disadvantageous, because it delays or even prevents implicit learning of the intended task (see, e.g., Baddeley \& Wilson, 1994; McClelland, 2001). The switch benefits in task switching can be viewed as an indicator of these negative effects of error learning.

From this perspective, the question arises of whether control processes exist that can counteract the negative effects of error learning. As we have shown, error detection alone does not prevent incidental learning (Steinhauser $\&$ Hübner, 2006). However, such detection might at least initiate processes that reduce the negative effects of error learning on subsequent performance. Indeed, a kind of error-induced control that might be relevant in this respect has been reported by Ridderinkhof (2002).

Ridderinkhof (2002) examined the effects of errors on subsequent performance in a Simon paradigm. In this paradigm, one typically observes that an irrelevant spatial stimulus attribute interferes with performance, depending on whether the spatial stimulus attribute is compatible or incompatible with a spatial response attribute (for a review, see Lu \& Proctor, 1995). Ridderinkhof found that the interference from the irrelevant spatial attribute was reduced on trials following errors. However, this effect was restricted to posterror trials with relatively long response times (RTs). Posterror trials with fast responses showed no reduction in interference.

He explained this result by assuming that interference emerges because the irrelevant spatial stimulus attribute activates the corresponding response via an automatic processing route (see, e.g., Kornblum, Hasbroucq, \& Osman, 1990). If an error is caused in this way, the system tries to reduce the interference on posterror trials by selectively inhibiting the automatic route immediately after onset of the new stimulus. Because inhibition requires some time to build up, the corresponding reduction of interference is effective only on posterror trials with a relatively slow response. Fast responses, on the other hand, are triggered before inhibition takes place (see also Bub, Masson, \& Lalonde, 2006; Ridderinkhof, Scheres, Oosterlaan, \& Sergeant, 2005; Ridderinkhof, van den Wildenberg, Wijnen, \& Burle, 2004).

In the present study, we examined whether an analogous process can counteract the negative effects of strengthening the wrong task. Assume that a task $A$ is required on trial $n-1$, but the erroneous application of a task $B$ caused an error on this trial. Because this led to the strengthening of task $B$, we would expect a benefit for "switching" to this task on trial $n$, and this is just what we observed in our earlier study. Now assume, however, that detecting the error on trial $n-1$ leads to a task-set adjustment that implies that the next stimulus will trigger the inhibition of the erroneously strengthened rules for task $B$ on the following trial $n$. Moreover, this inhibition takes place irrespective of whether task $A$ or $B$ is required on trial $n$. In this case, performing task $B$ on trial $n$ would no longer be beneficial. Rather, the inhibition could even be so strong that "switching" to this task would even be costly. Thus, such a mechanism would completely compensate for the effects of erroneous task strengthening. As a result, switch effects following errors would equal those following correct responses.

One might ask, at this point, if such error-induced inhibition exists, why do we observe strong switch benefits following errors at all? This could be explained by Ridderinkhof's (2002) idea that inhibition on trial $n$ takes time to build up after it is triggered by the stimulus. As a consequence, its compensatory effects should be observable only for slow responses. In our earlier experiments (Steinhauser \& Hübner, 2006), we considered only very fast responses, because we employed a deadline procedure - that is, the participants had to respond before an acoustical deadline signal. This method produces high error rates, which is necessary for analyzing posterror effects. However, it also reduces RTs. Under these conditions, it is rather unlikely that effects of a slow inhibitory process would be observed.

\section{Experimental Approach}

In the following sections, we report three experiments designed to test the idea that the effects of erroneous task strengthening are compensated for on trials with slow re- 
sponses. In Experiment 1, we modified the paradigm of Steinhauser and Hübner (2006). Instead of inducing errors by means of a deadline procedure, we simply instructed the participants to respond quickly. This led to many errors, but also allowed for occasional slow responses. In Experiment 2, we applied a paradigm in which each trial consisted of two tasks, in which a deadline was used for the first task only. In this way, many errors were obtained for the first task, but many slow responses were obtained for the second task (for which the switch effects were measured). Finally, in Experiment 3, we tested an alternative explanation by examining whether our effects depended on the interval between two trials.

\section{EXPERIMENT 1}

The first experiment was similar to those in Steinhauser and Hübner (2006). The participants alternated randomly between magnitude (less/greater than 5) and parity judgments (odd/even), and the relevant task was indicated by a cue on each trial. However, we did not employ a deadline paradigm as in our earlier experiments. We simply encouraged our participants to respond quickly. In this way, we hoped to obtain many errors without preventing occasional slow responses. We predicted switch benefits for fast responses on posterror trials but no switch benefits, or even switch costs, for slow responses in such trials. A distributional analysis was performed to test these predictions.

Two methodological aspects of our experiment are different from those usually found in other studies. First, we used only incongruent stimuli - that is, stimuli that required different responses for each task. This was crucial for obtaining switch benefits, because errors on congruent stimuli cannot be due to task confusion (for discussions, see Experiment 2 and Steinhauser \& Hübner, 2006). Second, to exclude the possibility that our results were due to strengthening of the stimulus-response rules, stimulus repetitions were excluded from the analyses.

\section{Method}

Participants. Fifteen participants ( 12 female, 3 male), from 19 to 38 years of age (mean 25.2) and with normal or corrected-to-normal vision, participated in the study. The participants were recruited at the Universität Konstanz and were paid $€ 5 / \mathrm{h}$.

Apparatus. The stimuli were presented on a 21-in. color monitor. An IBM-compatible PC controlled stimulus presentation and response registration.

Stimuli. The stimuli were the digits $1,3,6$, and 8 , which spanned a width of $1.24^{\circ}$ and a height of $1.77^{\circ}$ of visual angle at a viewing distance of $127 \mathrm{~cm}$. A circle and a square, both $1.43^{\circ}$ in diameter, were used as cues. The cues and stimuli were presented in white on a black background.

Design and Procedure. On each trial, participants had to apply one of two judgments to a digit presented on the screen. In the parity judgment, the digit had to be classified as odd or even. In the magnitude judgment, it had to be classified as less than or greater than 5 . Responses were given by pressing a response button with the index finger (even, less than 5) or the middle finger (odd, greater than 5) of the right hand. Each trial started with the presentation of the cue for $300 \mathrm{msec}$, followed by a blank screen for $900 \mathrm{msec}$. The stimulus was presented for $150 \mathrm{msec}$ and was followed by a blank screen. $1,000 \mathrm{msec}$ after the response, a new trial started. No feedback on the accuracy of the response was provided.
Participants worked through 26 blocks of 64 trials, resulting in a total of 1,664 trials. Within each block, the order of judgments was randomized. The blocks were distributed across three experimental sessions $(6 / 10 / 10)$ that took place on separate days. The first session started with 4 practice blocks. After each practice block, the participants received verbal feedback whenever the error rate in the block was below $15 \%$, in which case they were encouraged to respond more quickly.

\section{Results}

Trials on which the previous stimulus was repeated $(25 \%)$ and trials following spontaneously corrected errors $(<1 \%)$ were excluded from the analysis. We first report the mean results for RTs and error rates and then the results of the distributional analyses.

Mean results. Each trial was classified with respect to the variables task (parity, magnitude), task transition (task repetition, task switch), accuracy on trial $n-1$ (correct, error), and response transition (response repetition, response switch). RTs of correct responses and error rates were entered into a four-way ANOVA with repeated measurements on each of these variables. By including the variables task and response transition, we ensured that each type of response transition as well as each task contributed equally to each cell mean. In the following discussion, however, these variables are not considered further.

For the RT data, the analysis revealed a significant main effect of accuracy on trial $n-1[F(1,14)=4.80, p<.05]$, as well as a marginally significant interaction between accuracy on trial $n-1$ and task transition $[F(1,14)=3.56$, $p=.08]$. Whereas switch costs occurred after a correct response (task repetition, $442 \mathrm{msec}$; task switch, $464 \mathrm{msec}$ ), no switch effect emerged after an error (task repetition, $467 \mathrm{msec}$; task switch, $467 \mathrm{msec}$ ). Similarly, the error rates revealed a significant main effect of accuracy on trial $n-1$ $[F(1,14)=4.83, p<.05]$, as well as a significant interaction between accuracy on trial $n-1$ and task transition $[F(1,14)=4.90, p<.05]$. Correct responses produced switch costs on the subsequent trial (task repetition, $13.8 \%$; task switch, $16.3 \%$ ), whereas errors produced switch benefits (task repetition, 20.0\%; task switch, 16.7\%).

Distributional analysis. To estimate the effects of our variables for different regions of the RT distribution, we applied the following method. For each condition - resulting from the combination of the variables task, task transition, accuracy on trial $n-1$, and response transition - trials were rank-ordered according to RT and separated into four quantiles. ${ }^{2}$ In this way, each trial was assigned to a certain level of the variable quantile $(25 \%, 50 \%, 75 \%, 100 \%)$. The mean RTs for the four quantiles were $318 \mathrm{msec}, 392 \mathrm{msec}$, $457 \mathrm{msec}$, and $643 \mathrm{msec}$, respectively.

Figure 1 shows the interaction between accuracy on trial $n-1$ and task transition in the error rates and the RTs of correct responses, separately for each quantile. For the error rates (left panel), we observed the typical pattern (switch costs following correct responses but switch benefits following errors) for the first three quantiles. As expected, however, the results were different for the quantile representing the slowest responses. Here, switch costs emerged after errors that were similar to those after correct responses. 


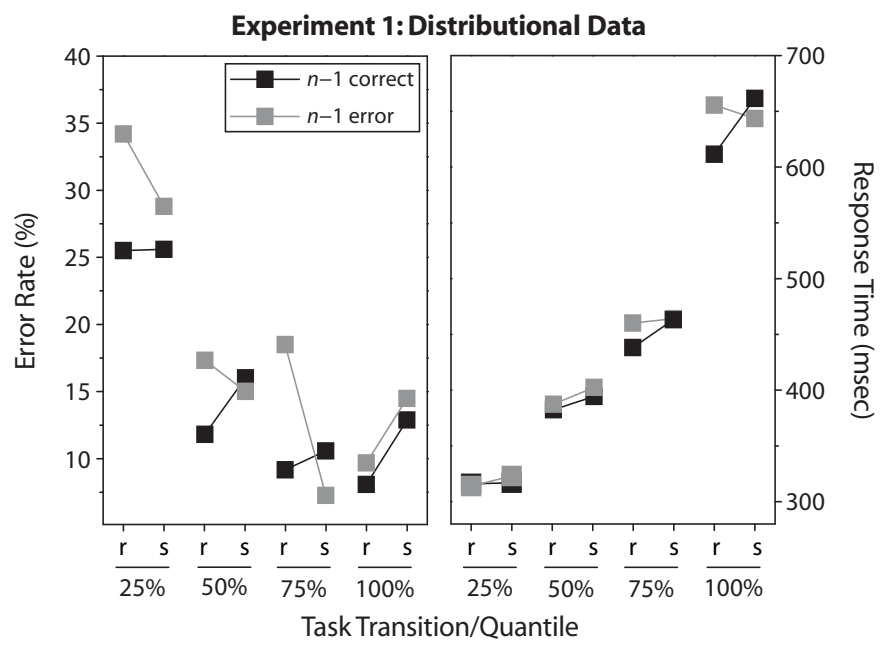

Figure 1. Experiment 1: Mean response times of correct responses (right panel) and error rates (left panel) as a function of accuracy on trial $n-1(n-1$ correct, $n-1$ error), task transition (repetition, switch), and quantile $(25 \%$, $50 \%, 75 \%, 100 \%$ ). r, repetition; s, switch.

To test our hypothesis that switch effects following errors are different between slow and fast responses, we applied a method similar to that of Ridderinkhof (2002). For each pair of adjacent quantiles, $Q_{n}$ and $Q_{n+1}$, we tested whether the pattern of switch effects changed significantly. In this way, each test corresponded to the interaction between task transition (repetition, switch) and quantile $\left(Q_{n}, Q_{n+1}\right)$. As a result, the switch effects did not differ between the first and second $(F<1)$ or the second and third $(F<2.5)$ quantiles. However, there was a significant difference between the switch effects in the third and fourth quantiles $[F(1,14)=$ $10.5, p<.01]$. To ensure that this significant effect was not due only to the relatively strong switch benefits in the third quantile, we also computed the contrast between the switch effect in quantile four and the averaged switch effects from the first three quantiles, which reached significance as well $[F(1,14)=7.63, p<.05]$. In contrast, the same analyses revealed no significant differences in the RT data. However, Figure 1 (right panel) shows that both switch costs and switch benefits for RTs increased with increasing RT.

\section{Discussion}

The goal of Experiment 1 was to examine whether switch benefits following an error are absent on trials with slow responses. To obtain a sufficient number of errors as well as a sufficient number of slow responses, the participants were instructed to respond quickly, but no deadline was applied. As expected, the overall error rate $(16.7 \%)$ was relatively large. Moreover, the mean RT (458 $\mathrm{msec}$ ) was substantially longer than those in our previous deadline experiments (e.g., $335 \mathrm{msec}$; Steinhauser \& Hübner, 2006, Experiment 3). The analysis of mean performance replicated our earlier results: Switch costs occurred after correct responses, whereas switch benefits occurred after errors.

A more detailed result, however, was obtained via the distributional analysis. It revealed different switch ef- fects for fast and slow responses. Whereas fast responses showed switch benefits following errors, responses in the slowest quantile not only showed no switch benefits, but actual switch costs. However, this outcome was restricted to the analysis of error rates. For RTs, switch benefits occurred in all quantiles. ${ }^{3}$ At the moment, it remains unclear why we observed the supposed effect of error-induced inhibition only in the error rates. However, we will discuss this issue in a later section.

Altogether, our results support the idea that the task that caused the error on trial $n-1$ is inhibited on trial $n$. Because this inhibition starts at stimulus onset and requires some time to become effective, only slow responses benefit from it. On trials with such responses, the effect of the erroneously strengthened task is not only reduced (which would lead to a reduced switch benefit) but turns into a switch cost. Before discussing further implications of this result, we will report further experiments in which we replicated the result using different methods.

\section{EXPERIMENT 2}

In Experiment 1, the error-induced compensatory effect was substantial only for the slowest $25 \%$ of the responses. This could have been due to the fact that we employed a method that still induced only few slow responses. Therefore, we used a different method in Experiment 2. On each trial, the participants had to perform two tasks. Whereas the type of the first task was always indicated by a cue, the type of the second task was either the same as or different from the first one, depending on whether the participant was performing in a task-repetition or task-switch block. Crucially, a deadline procedure was used for the first task but not for the second. In this way, a high error rate should be produced for the first task as well as a relatively large proportion of slow responses for the second, for which the 
switch effects were computed. Although we did not expect participants to always switch from fast and error-prone first-task performance to slow and accurate second-task performance, we at least hoped to obtain a larger number of slow responses than in the previous experiment.

Unfortunately, this procedure is not unproblematic, because switch and repetition trials differed with respect to the number of tasks that were relevant in a trial. Since only one task was relevant on a repetition trial but two tasks were relevant on a switch trial, this alone could produce large switch costs. In fact, such an effect would be more similar to so-called mixing costs, which refer to the impaired performance on mixed-task blocks (in which two or more tasks are relevant) relative to single-task blocks (in which only one task is relevant), and which have been attributed to the increased need to control task conflicts (see, e.g., Rubin \& Meiran, 2005; Steinhauser \& Hübner, 2005). However, such costs should affect performance after both errors and correct responses in the same way. Moreover, since mixing costs are typically obtained in RTs only (see Rubin \& Meiran, 2005; Steinhauser \& Hübner, 2005), we still could hope to find substantial effects in the error rates, similar to those in Experiment 1.

Because we introduced a new procedure, we also wanted to replicate a crucial result from our previous study (Steinhauser \& Hübner, 2006). There, we showed that switch benefits occurred only after errors for incongruent stimuli- that is, for stimuli associated with different responses for each task. In contrast, no switch benefits were obtained after errors for congruent stimuli - those associated with the same response for each task. This result is crucial for our assumption that switch benefits result from task confusion in the previous trial. Since task confusions cannot cause an error for congruent stimuli, similar numbers of task confusions should underlie errors and correct responses on congruent stimuli. As a consequence, correct responses and errors for congruent stimuli cannot cause different switch effects on the subsequent trial, provided that only task confusions produce subsequent switch benefits. As a consequence, we predicted that switch benefits should be obtained only following errors on incongruent stimuli. We tested this hypothesis by also including congruent stimuli.

\section{Method}

Participants. Twelve participants ( 8 female, 4 male), from 19 to 30 years of age (mean 23.0) and with normal or corrected-to-normal vision, participated in the study. The participants were recruited at the Universität Konstanz and were paid $€ 5 / \mathrm{h}$.

Apparatus. The stimuli were presented on a 21-in. color monitor. An IBM-compatible PC controlled stimulus presentation and response registration.

Stimuli. The stimuli and cues were the same as in Experiment 1, with the exception that now all the digits from 2 to 9 were used. In this way, half of the numerals (e.g., the numeral 2) were congruentthat is, the same response was required for each task - whereas the other half (e.g., 3) were incongruent - that is, different responses were required for each task.

Design and Procedure. On each trial, participants had to classify two serially presented digits according to the same judgment rule or to two different ones. Parity and magnitude judgments were used, and responses were given by pressing a response button with the index finger (even, less than or equal to 5) or the middle finger (odd, greater than 5) of the right hand. Each trial started with the presentation of the cue for $1,000 \mathrm{msec}$, followed by a blank screen for $1,000 \mathrm{msec}$. The first stimulus was presented for $250 \mathrm{msec}$ and was followed by a blank screen. The participants had to apply the task indicated by a cue (circle $=$ parity, square $=$ magnitude). After a specific (deadline) interval, an acoustical deadline signal was presented for $150 \mathrm{msec}$. 1,500 $\mathrm{msec}$ after the first response, the second stimulus was presented for $250 \mathrm{msec}$. A new trial started $1,500 \mathrm{msec}$ after the second response. No error feedback was provided. The participants were instructed to respond before the deadline signal on the first task, but on the second task, they were instructed only to respond as quickly and accurately as possible.

A block consisted of 64 trials, which resulted from the combination of each of the eight numerals as the first and the second stimulus. Participants worked through 16 blocks, resulting in a total of 1,024 trials. The blocks were distributed across two experimental sessions. In half of the blocks, the participants were instructed to switch the task on the second stimulus. In the other half, they had to perform the same task for both stimuli. Switch and repetition blocks alternated, whereas the type of the first block in each session was counterbalanced across participants and sessions. The two experimental sessions were preceded by a practice session, in which the deadline interval was individually determined. The first 4 blocks of this session were without deadline, and a deadline was introduced in the last 4 . The deadline interval for the whole experiment was set to the mean of the two medians of Blocks 7 and 8, at the end of the practice session.

\section{Results}

Trials with stimulus repetition (12.5\%) as well as those following spontaneously corrected errors $(<1 \%)$ were excluded from the analysis. Although we were mainly interested in the performance for the second task, for completeness, we also report the results for the first task.

Mean performance for Task 1. RTs of correct responses and error rates of the first task were analyzed by two-way ANOVAs with repeated measurements for the variables task transition (task repetition, task switch) and Stimulus 1 congruency (congruent, incongruent). With respect to RTs, responses were slower for incongruent stimuli $(385 \mathrm{msec})$ than for congruent stimuli $(370 \mathrm{msec})$ $[F(1,11)=9.31, p<.05]$. For error rates, a significant main effect of Stimulus 1 congruency $[F(1,11)=35.5, p<$ $.001]$ was qualified by a significant interaction between task transition and Stimulus 1 congruency $[F(1,11)=$ $11.9, p<.01]$. In task-repetition blocks, more errors were produced for incongruent (33.3\%) than for congruent $(15.4 \%)$ stimuli. However, this difference was reduced in task-switch blocks (30.6\% vs. $17.6 \%$, respectively).

Mean performance for Task 2. RTs of correct responses and error rates of the second task were analyzed by four-way ANOVAs with repeated measurements for the variables task transition (task repetition, task switch), accuracy on Task 1 (correct, error), Stimulus 1 congruency (congruent, incongruent), and Stimulus 2 congruency (congruent, incongruent). We report only significant effects that were not qualified by greater interactions.

For error rates, the four-way interaction of the variables was significant $[F(1,11)=5.83, p<.05]$. The corresponding data are depicted in Figure 2. To examine this interaction in detail, we calculated the contrast representing the interaction between task transition and accuracy in Task 1 separately for each combination of the two congruency variables. It turned out that this contrast reached signifi- 
cance only when the stimuli for both tasks were incongruent $[F(1,11)=14.5, p<.01]$. For all other combinations, the contrasts were far from significant $(F \mathrm{~s}<1)$.

For RTs, no effect involving the interaction between task transition and accuracy in Task 1 was reliable. Rather, there was a significant main effect of task transition $[F(1,11)=13.0, p<.01]$, indicating that RTs were generally higher on task-switch trials $(614 \mathrm{msec})$ than on task-repetition trials $(528 \mathrm{msec})$. Furthermore, there was a significant main effect of accuracy in Task $1[F(1,11)=$ $8.76, p<.05]$, indicating that RTs were increased after an error $(599 \mathrm{msec})$ relative to after a correct response $(543 \mathrm{msec})$. Finally, there was a significant main effect of Stimulus 2 congruency $[F(1,11)=27.8, p<.001]$, indicating that RTs were slower for incongruent $(609 \mathrm{msec})$ than for congruent $(533 \mathrm{msec})$ stimuli.

Distributional analysis of Task 2 performance. Our analysis revealed large switch costs for RTs. These effects were similar, irrespective of whether an error or a correct response had been produced for Task 1. Moreover, the effects were also similar irrespective of whether the first stimulus was congruent or incongruent. From this, we concluded that the RTs reflected differences between taskrepetition and task-switch trials that were more related to mixing costs than to the type of switch costs observed in Experiment 1. Accordingly, our distribution analysis focused on the error rates, whereas the RTs were analyzed only to exclude a speed-accuracy trade-off.

For the error rates, reliable switch effects were obtained only for trials in which both stimuli were incongruent.
Therefore, we restricted our distributional analyses to this case. For each remaining condition, resulting from the combination of the variables task transition and accuracy on Task 1, trials were rank-ordered according to RT and classified into three quantiles. ${ }^{4}$ Unlike in Experiment 1, we used three instead of four quantiles, because we had fewer trials in the conditions of interest. However, four quantiles would have produced qualitatively similar results. Each trial was assigned to one level of the variable quantile $(33 \%, 66 \%, 100 \%)$. The mean RTs for the three quantiles were $438 \mathrm{msec}, 569 \mathrm{msec}$, and $809 \mathrm{msec}$, respectively.

Figure 3 shows the interaction between accuracy on Task 1 and task transition in the error rates (left panel) and the RTs (right panel), separately for each quantile. As can be seen in the error rates, there were switch costs after a correct response for each quantile. However, switch benefits after errors were observed only for the fastest responses. Whereas the switch benefits already turned into switch costs for the second quantile, these costs were slightly increased for the third quantile. This conclusion received support when we calculated the planned contrast representing the two-way interaction between task transition (repetition, switch) and quantile $\left(Q_{n}, Q_{n+1}\right)$ for trials with an error in the first task. It turned out that the switch effects already differed significantly between the first and second quantiles $[F(1,11)=$ $4.80, p<.05]$. In contrast, the difference between the second and third quantiles did not reach significance $(F<1)$. With respect to the RTs, no effect of quantile on the switch effect was revealed. Rather, we obtained consistently large switch costs following errors in each quantile.

Experiment 2: Mean Data

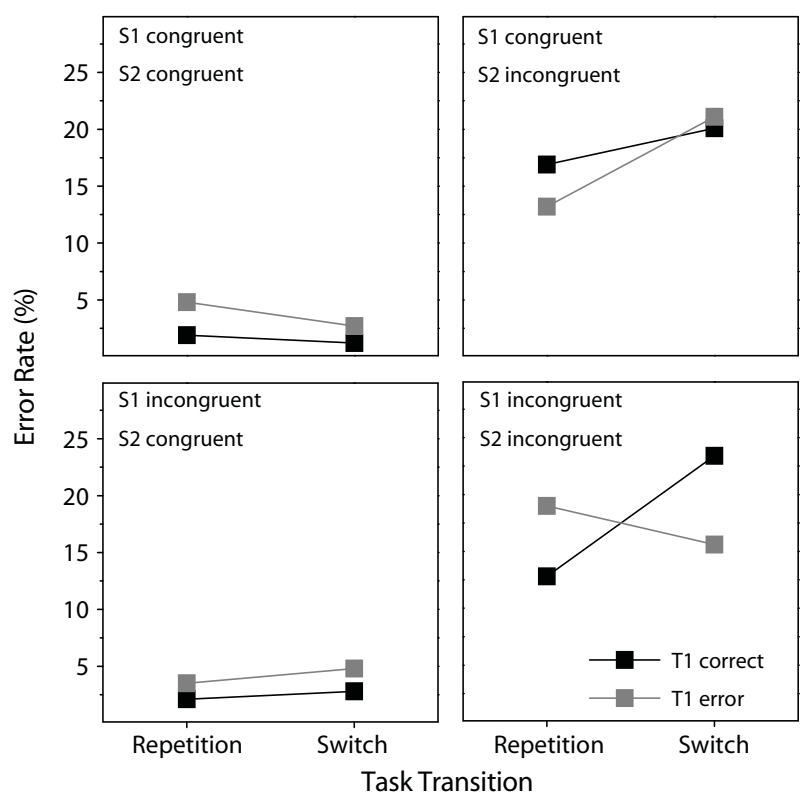

Figure 2. Experiment 2: Mean error rates of Task 2 as a function of accuracy on Task 1 (correct, error), task transition (repetition, switch), S1 congruency (congruent, incongruent), and S2 congruency (congruent, incongruent). S1, Stimulus 1; S2, Stimulus 2; T1, Task 1. 


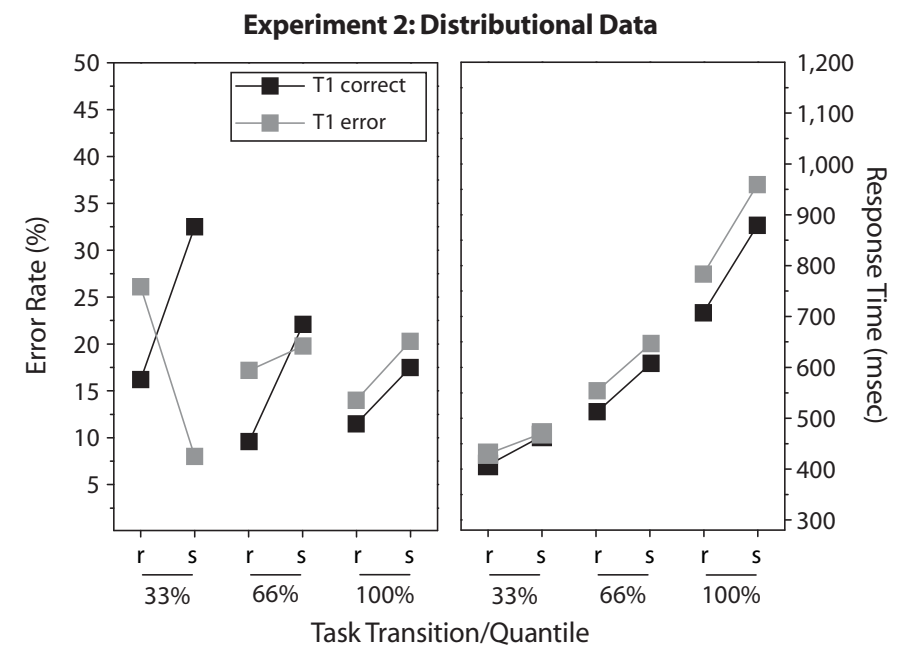

Figure 3. Experiment 2: Mean response times of correct responses (right panel) and error rates (left panel) of Task 2 as a function of accuracy on Task 1 (correct, error), task transition (repetition, switch), and quantile $(33 \%, 66 \%$, $100 \%$ ). T1, Task 1; r, repetition; s, switch.

\section{Discussion}

The main purpose of Experiment 2 was to replicate the results of our first experiment by using a new paradigm. Here, each trial consisted of two tasks. A deadline was present for Task 1 but not for Task 2, and Task 2 performance was used to calculate switch costs. Our intention was to obtain many errors for Task 1 and relatively slow responses for Task 2. Indeed, if we consider trials on which both stimuli were incongruent, the Task 1 error rate $(31.9 \%)$ was considerably higher than the error rate in Experiment 1 (16.7\%). Also, the mean RT for Task 2 on these trials was much higher $(607 \mathrm{msec})$ than that in Experiment $1(458 \mathrm{msec})$. The error rate for Task 2 on these trials $(17.7 \%)$ was similar to that in Experiment 1.

As expected, responses were generally slower on switch trials than on repetition trials, presumably because two tasks were relevant on switch trials, whereas only one task was relevant on repetition trials. In this way, RTs on switch trials were prolonged, reflecting a phenomenon similar to so-called mixing costs (see, e.g., Rubin \& Meiran, 2005; Steinhauser \& Hübner, 2005). Such an effect did not occur for Task 1, which is not surprising, because the deadline applied in this task was similar for both switch and repetition trials. ${ }^{5}$ In any case, because of this pattern of RT results, we restrict our discussion to the error rates.

The error rates largely replicated our earlier results. In the mean data, we found substantial switch benefits following errors, but switch costs following correct responses. These effects, however, were pronounced only for incongruent stimuli in Task 2, which might have resulted from the fact that the error rate was rather low for congruent stimuli. This interpretation is also in line with other results showing that switch effects are typically smaller for congruent stimuli (e.g., Meiran, 2000). Switch benefits were found exclusively on trials with Task 1 errors for incongruent stimuli. On trials with a congruent first stimulus, errors were followed by even higher switch costs than those of correct responses. This replicates the results of Steinhauser and Hübner (2006) and shows that only errors resulting from task confusions cause subsequent switch benefits.

Most importantly, the distributional analysis revealed strong switch benefits for fast responses after an error, as in Experiment 1. In contrast, slow responses after an error showed switch costs. Unlike in Experiment 1, though, the transition from benefits to costs had already occurred on trials with intermediate RTs, which confirmed our expectation. The greater the number of slow responses, the more trials (or quantiles) should show switch costs following errors.

\section{EXPERIMENT 3}

With respect to error rates, our first two experiments revealed a consistent pattern. Following errors on incongruent stimuli, we observed switch benefits on trials with fast responses, but switch costs on trials with slow responses. We assume that this pattern reflects the net effect of two processes: strengthening of task errors, which affects all posterror trials, as well as error-induced inhibition, which compensates for the effect of error strengthening on posterror trials with slow responses. Unfortunately, however, fast and slow responses also differ in a way that allows for an alternative interpretation; more specifically, the interval between the responses on trial $n$ and trial $n-1$ is longer for slow than for fast trial- $n$ responses. If we assume that the effect of error strengthening is transient and decays over time, this could explain why switch benefits are observed on trials with fast responses only. The switch costs on trials with slow responses could be related to other processes, such as task preparation (see, e.g., De Jong, 2000).

To test this idea, we conducted a further experiment in which we manipulated the response-cue interval (RCI)that is, the interval between the response on one trial 
and the cue on the following trial. Using the paradigm from Experiment 1, we established two RCIs: 1,000 and $2,000 \mathrm{msec}$. For the short RCI, we expected results similar to those in Experiment 1, in which the same RCI was applied. However, a different picture could emerge when the $\mathrm{RCI}$ was prolonged. If the effect of error strengthening decays over time, a longer RCI should lead to reduced or no switch benefits on trials with fast responses. In contrast, the prediction of our error-induced inhibition hypothesis is less clear for this condition. If switch benefits are due to a strengthening mechanism that is related to procedural learning, they should be stable over time, at least for the intervals used in this experiment. Therefore, we would expect switch benefits for fast responses, even with a long RCI. However, it is unclear whether a long RCI would affect the error-induced inhibition process and, therefore, the switch costs on trials with slow responses.

\section{Method}

Twelve participants ( 11 female, 1 male) from 20 to 26 years of age (mean 21.7) with normal or corrected-to-normal vision participated in the study. Participants were recruited at the Universität Konstanz and were paid $€ 5 / \mathrm{h}$.

The stimuli, tasks, and procedure were the same as in Experiment 1 , with one exception. In half of the blocks, the interval between the response and the onset of the following cue (the RCI) was $1,000 \mathrm{msec}$, as in Experiment 1. In the other half, an RCI of $2,000 \mathrm{msec}$ was used instead. The two block types alternated in the test sessions as well as in the practice session. The RCI of the first block was counterbalanced across participants.

\section{Results}

Again, trials with stimulus repetitions $(25 \%)$ and trials following corrected errors $(<1 \%)$ were excluded from analysis.

Mean results. Error rates and RTs of correct responses were entered into a three-way ANOVA with the variables RCI (short, long), accuracy on trial $n-1$ (correct, error), and task transition (task repetition, task switch). For the RTs, only a significant effect of task transition was obtained $[F(1,11)=7.48, p<.05]$, indicating higher RTs on task-switch $(522 \mathrm{msec})$ than on task-repetition $(489 \mathrm{msec})$ trials. Interestingly, RTs were longer with a long RCI $(522 \mathrm{msec})$ than with a short RCI $(490 \mathrm{msec})$, although this effect failed to reach significance $(F<2.6)$. For the error rates, we obtained a significant interaction between accuracy on trial $n-1$ and task transition $[F(1,11)=26.5$, $p<.001]$. Whereas we observed switch costs following correct responses (task repetition, 5.8\%; task switch, $12.2 \%$ ), there were switch benefits following errors (task repetition, 10.4\%; task switch, 5.4\%).

Distributional analysis. To analyze the effects in the RT distributions, trials were rank-ordered according to RT separately for the variables RCI, task transition, and accuracy on trial $n-1.6$ Since each condition contained only half of the trials it had in Experiment 1, we now used two quantiles only. Accordingly, each trial was assigned to one level of the variable quantile $(50 \%, 100 \%)$. The mean RTs for the two quantiles were 383 and $634 \mathrm{msec}$, respectively.

Figure 4 shows the interaction between RCI, task transition, and accuracy on trial $n-1$ for error rates (left panels) and RTs (right panels). Inspection of the error rates reveals that both RCI conditions show switch benefits following errors in the first quantile. However, whereas switch costs following errors are apparent in the second quantile of the short-RCI condition, this is not the case in the second quantile of the long-RCI condition. This was confirmed when we analyzed the error rates of posterror trials in a three-way ANOVA with repeated measurements on the variables RCI (short, long), task transition (task repetition, task switch), and quantile $(50 \%, 100 \%)$. The ANOVA revealed a significant interaction between all three variables $[F(1,11)=11.4, p<.01]$. Moreover, planned contrasts showed that task transition interacted with quantile for the short-RCI condition $[F(1,11)=20.6, p<.001]$ but not for the long-RCI condition $(F<1)$.

For RTs, we observed no switch effect on posterror trials in the first quantile of both RCI conditions. In the second quantile, we obtained switch costs on posterror trials that were smaller in the long-RCI condition than in the short-RCI condition. Thus, the pattern in the RTs roughly mirrored that in the error rates. However, none of these effects reached significance $(F<1)$.

\section{Discussion}

Experiment 3 was designed to examine how switch effects on posterror trials are affected by the interval between two trials. To achieve this, we manipulated the interval between the response and the subsequent cue. Again, the effects were found mainly in the error rates. In contrast to the previous experiments, however, the RTs showed a pattern roughly similar to that in the error rates, although these effects did not reach significance. Therefore, our discussion focuses on the error rates. The short-RCI condition was identical to the task in Experiment 1. Accordingly, it is not surprising that in this condition we observed switch benefits on posterror trials with fast responses but switch costs with slow responses. The crucial question is now whether this pattern changed in the long-RCI condition. Indeed, such a change was observed, though in a rather unexpected way. For posterror trials with fast responses, we observed similar switch benefits in both RCI conditions. However, the switch costs on posterror trials with slow responses disappeared when the RCI was long. Such a pattern is clearly inconsistent with the idea that the effects of error strengthening decay. Such a hypothesis would predict switch costs on posterror trials with fast responses when the $\mathrm{RCI}$ is long.

In contrast, our data suggest a different interpretation. First, they demonstrate, as expected, that the effects of error strengthening are stable over time. Neither the switch costs following correct responses nor the switch benefits following errors were reduced when the RCI was prolonged. This confirms earlier results (e.g., Altmann, 2005; Meiran, Chorev, \& Sapir, 2000) and supports the idea that switch benefits are related to procedural learning in long-term memory (Steinhauser \& Hübner, 2006). Second, in contrast to error learning, error-induced inhibition seems to be sensitive to the intertrial interval. This is not implausible, given how error-induced inhibition presumably works. Similar to Ridderinkhof (2002), we 
Experiment 3: Distributional Data

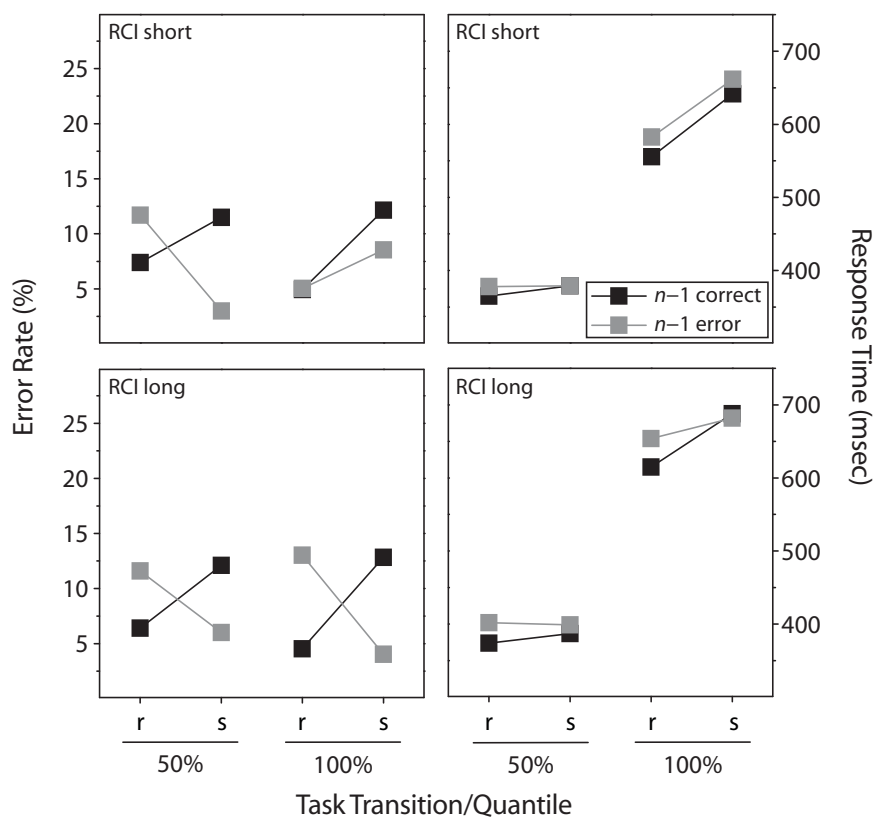

Figure 4. Experiment 3: Mean response times of correct responses (right panel) and error rates (left panel) as a function of RCI (short, long), accuracy on trial $n-1$ (correct, error), task transition (repetition, switch), and quantile $(50 \%, 100 \%)$. RCI, response-cue interval; r, repetition; s, switch.

suggest that error detection does not directly trigger inhibition. Rather, error detection adjusts the task set in a way that makes the next stimulus trigger the inhibition of the previously strengthened task. Otherwise, one cannot explain why the effectiveness of inhibition increases with RTs on trial $n$. Our results could imply that this task-set adjustment is transient. With an RCI of 2 sec (and a cuestimulus interval of another second), the system might be unable to maintain this adjustment. One reason could be that the long intertrial period reduces participants' arousal and makes them susceptible to internal distraction. This could also explain why the long RCI did not improve overall performance, but even tended to increase RTs. Such conditions are well-suited to impair endogenous control, whereas effects of associative learning remain unaffected. According to this view, the present results fully confirm our interpretation of switch effects following errors.

\section{GENERAL DISCUSSION}

The goal of the present study was to investigate whether mechanisms can counteract the negative effects of error learning. The effects of error learning were measured by switch benefits in a task-switching paradigm, which indicated erroneous task strengthening (Steinhauser \& Hübner, 2006). A specific mechanism that has the potential to reduce the negative consequences of error learning is error-induced inhibition, which has been proposed by Ridderinkhof (2002). He showed that automatic processes in a
Simon task are selectively inhibited when these processes caused an error on the preceding trial. However, because he observed the effect only for slow responses on trial $n$, Ridderinkhof concluded that the inhibition requires some time to become effective.

Here, we analogously hypothesized that a task is selectively inhibited on trial $n$ when it caused an error on trial $n-1$, irrespective of which task is actually relevant on trial $n$. Moreover, because we also assumed that the inhibition requires some time, we predicted that following an error on trial $n-1$, fast responses on trial $n$ would show a switch benefit (indicating erroneous task strengthening on trial $n-1)$, whereas slow responses on trial $n$ would show switch costs (indicating error-induced inhibition on trial $n$ of the previously strengthened task).

This prediction was tested in three experiments, in which different variants of the task-switching paradigm were applied. In Experiment 1, the deadline procedure from our former experiments was replaced with the simple instruction to respond quickly. In this way, we should be able to obtain enough errors, yet also occasional slow responses. As predicted, we found switch costs for the slowest $25 \%$ of trials following errors, but switch benefits for the remaining trials. However, this held only for error rates.

In Experiment 2, we used a paradigm in which two tasks had to be performed on each trial. A deadline for Task 1 was intended to increase the error rate for this task. In contrast, no such deadline was applied for Task 2, for which we computed switch effects. Indeed, as intended, 
we obtained much slower responses for Task 2 than in Experiment 1. Moreover, switch costs following errors were obtained for even more trials - that is, for about the slowest half of the responses. In addition, this experiment also replicated the finding from Steinhauser and Hübner (2006) that switch benefits occur only following errors for incongruent stimuli. Again, these effects were obtained only for error rates, whereas the RTs showed constantly high switch costs. However, this finding could be attributed to mixing costs due to an increased number of relevant tasks on task-switch trials.

Finally, in Experiment 3, we manipulated the RCIthat is, the interval between a response and the subsequent cue - in order to exclude an alternative explanation related to the decay of switch benefits. This experiment not only replicated the results of Experiment 1 for a normal RCI, it also demonstrated that, whereas the switch benefits on trials with fast responses remained unaffected by RCI, the switch costs on trials with slow responses disappeared when the RCI was prolonged. This pattern supports the ideas that switch benefits reflect the long-lasting effect of error learning but that the reinstatement of switch costs after an error relies on a more susceptible endogenous control process.

Taken together, the present results and those of an earlier study (Steinhauser \& Hübner, 2006) provide evidence for two mechanisms. First, performance on trial $n$ reflects task strengthening on trial $n-1$. Whether this process results in switch costs or switch benefits depends on whether the intended task had actually been applied on trial $n-1$. Committing an error on trial $n-1$ because of unintentional application of the wrong task, and leaving this error uncorrected, produces switch benefits on trial $n$, at least for fast responses. Otherwise, switch costs are instead obtained on trial $n$. On the basis of earlier evidence (Philipp et al., 2007; Schuch \& Koch, 2003; Steinhauser \& Hübner, 2006), we suggest that task strengthening reflects a strengthening of category-response rules that is triggered by an overt response. Moreover, this strengthening seems to be related to procedural learning, supported by the present finding that its effect is relatively long-lasting (see also Altmann, 2005; Meiran et al., 2000).

Second, performance on trial $n$ also reflects errorinduced inhibition on trial $n$. Whether this process leads to switch costs even after an error depends on whether the response on trial $n$ is sufficiently slow for the inhibition to become effective. This implies that inhibition is not triggered by error detection on trial $n-1$. Rather, we suggest that error detection induces a task-set adjustment, which implies that the subsequent stimulus will trigger inhibition of the previously executed task (see Ridderinkhof, 2002). This adjustment seems to be susceptible to decay or distraction. Taken together, erroneous task strengthening reflects an incidental, unsupervised learning mechanism, whereas error-induced inhibition reflects a method of compensating for the negative effect of this learning mechanism in case of an error.

Before we elaborate these ideas in more detail, one aspect of our data requires closer consideration. Whereas the effects of error-induced inhibition were observed in the error rates very consistently, the picture was more inconsistent in the RTs: In Experiment 1, we obtained switch benefits on posterror trials for each quantile. Because the strength of these switch benefits mirrored the switch costs in the corresponding quantiles on trials following correct responses, we suggested that this pattern reflects an increasing effect of task strengthening in the course of the distribution, as well as the absence of an effect of error-induced inhibition. In Experiment 2, we obtained the same strong switch costs in RTs following errors as following correct responses in each quantile. Here, we argued that the switch costs in RTs reflect mainly the increased number of relevant tasks on switch trials and the resulting mixing costs. Finally, in Experiment 3, we obtained a pattern in RTs following errors that roughly resembles the prediction of the error-induced inhibition account - that is, a transition from switch benefits to switch costs. However, in contrast to the error rates, this pattern was only slightly different for the two RCI conditions. Therefore, it is questionable whether the RT pattern really reflects the same process as the error rates.

When all of the results are taken together, it seems that error-induced inhibition mainly affects error rates. In contrast, switch effects in RTs are influenced only by task strengthening and other processes. In other words, inhibition of a task that caused an error in the preceding trial does not slow down execution of this task, but rather reduces the probability that this task will be erroneously executed once more. Unfortunately, we currently have no theory as to precisely how such a mechanism would work. Nevertheless, it seems plausible that error-induced inhibition affects mainly error rates - that is, the probability of task execution. This is best illustrated if we consider the case of a given task $A$ that is repeatedly practiced, while an alternative $\operatorname{task} B$ is potentially applicable. Under these conditions, erroneously strengthening task $B$ has two negative side effects: First, execution of task $A$ is slowed on the subsequent trial, and second, the probability is increased of again erroneously executing task $B$. The latter effect is the one that mainly threatens procedural learning of task $A$, because further task errors would lead to further erroneous task strengthening. In this way, a task error could initiate a chain reaction that might substantially impair task learning. In this view, it is reasonable that errorinduced inhibition should mainly affect the probability of task errors; simply prolonging the execution of the inhibited task would not be sufficient, because this would not prevent the inhibited task from again being strengthened.

\section{Further Considerations on Error-Induced Control}

The present results nicely complement those of Ridderinkhof (2002), who showed a similar effect in a different domain. Moreover, another phenomenon of errorinduced control fits with the present results. It has often been observed that responses following an error are slower but more accurate than those following a correct response (see, e.g., Fairweather, 1978; Laming, 1979; Rabbitt, 1968). This so-called posterror slowing (PES) has been interpreted as indicating the strategy of increasing the response criterion after an error, in order to reduce the risk of performing a further error. 
This raises the question of whether PES and errorinduced inhibition are related. On the one hand, PES could be an effective way to amplify the effect of error-induced inhibition. Because error-induced inhibition becomes effective only for slow responses, any measure that slows down responding will also improve the effectiveness of inhibition. Thus, one could speculate that an error triggers not only inhibition processes, but also PES in order to increase the effectiveness of inhibition. Indeed, if we compare the performance in our experiments, it is striking that in Experiment 2 we found not only greater switch costs following an error, but also more robust PES. On the other hand, error-induced inhibition could even contribute to PES. For instance, one could hypothesize that errorinduced inhibition captures central capacity and, in this way, slows down processing on trial $n$. This would produce PES, irrespective of whether the inhibited task or the notinhibited task is to be performed.

One might also ask whether error-induced inhibition is the only mechanism that can explain our data. Indeed, an alternative account might be derived from so-called dualprocess models. Such models typically assume that two processes compete for controlling the response: a more automatic process that is fast, inaccurate, parallel, and associative, and a more controlled process that is slow, accurate, serial, and algorithmic. The dual-process idea can be found in different but related domains (see, e.g., Hommel, 1998; Kornblum et al., 1990; Logan, 1988; Schneider \& Shiffrin, 1977; Steinhauser \& Hübner, 2005) and was used as a conceptual framework by Ridderinkhof (2002). Most importantly, dual-process models have been used to account for differential effects in the fast and slow tails of the RT distribution (e.g., De Jong, Liang, \& Lauber, 1994; Gratton, Coles, Sirevaag, Eriksen, \& Donchin, 1988; Vu $\&$ Proctor, 2004); slow responses are assumed to reflect the controlled process, whereas fast responses are thought to reflect the automatic process.

Correspondingly, one could hypothesize that switch costs following errors reflect the outcome of the slower, controlled processes, whereas switch benefits following errors reflect the outcome of the faster, automatic processes. To explain this, one could assume that controlled processing is based on top-down selection processes (Hübner, Futterer, \& Steinhauser, 2001; Steinhauser \& Hübner, 2005, 2007), which are particularly sensitive for the priming of task goals. Because of this, controlled processing could be strongly affected by the previously intended task. In contrast, automatic processing could be based on activation spreading along category-response associations whose strength is modified after task execution. As a consequence, automatic processing could be strongly affected by the previously applied task. In this way, after an error, controlled processing would produce switch costs, whereas automatic processing would produce switch benefits. An error could lead to an adjustment of the relative weights for the two pathways. Stronger controlled processing could then compensate for the negative effect of erroneous task strengthening, because it makes the output less sensitive to the strength of category-response associations. Although this account is rather speculative, it rep- resents a serious alternative to the notion of error-induced inhibition.

Finally, it might be interesting to note a further parallel between our results and others. In their seminal article, Baddeley and Wilson (1994) suggested that implicit memory can result in error learning in a stem completion task, whereas explicit memory serves to correct these errors. Basically, this theory mirrors our distinction between erroneous task strengthening and error-induced control in one crucial respect: Whereas error detection cannot prevent errors from being strengthened in implicit memory, the effects of error learning are compensated for by other processes. In the case of Baddeley and Wilson's paradigm, compensation occurs via explicit memory. In contrast, the same result is achieved by inhibition or controlled processing in our paradigm. Perhaps future research can reveal whether other parallels exist between these domains-for instance, the error detection process that coordinates both processes.

\section{AUTHOR NOTE}

This research was supported by Grant $\mathrm{Hu} 432 / 8$ to the second author from the Deutsche Forschungsgemeinschaft. We thank Iring Koch and two anonymous reviewers for helpful comments. Correspondence concerning this article should be addressed to M. Steinhauser, Universität Konstanz, Fachbereich Psychologie, Fach D29, D-78457 Konstanz, Germany (e-mail: marco.steinhauser(uni-konstanz.de).

\section{REFERENCES}

Allport, D. A., Styles, E. A., \& Hsieh, S. (1994). Shifting intentional set: Exploring the dynamic control of tasks. In C. Umiltà \& M. Moscovitch (Eds.), Attention and performance $X V$ : Conscious and nonconscious information processing (pp. 421-452). Cambridge, MA: MIT Press, Bradford Books.

Altmann, E. M. (2005). Repetition priming in task switching: Do the benefits dissipate? Psychonomic Bulletin \& Review, 12, 535-540.

Baddeley, A., \& Wilson, B. A. (1994). When implicit learning fails: Amnesia and the problem of error elimination. Neuropsychologia, 32, 53-68.

Bub, D. N., Masson, M. E. J., \& Lalonde, C. E. (2006). Cognitive control in children: Stroop interference and suppression of word reading. Psychological Science, 17, 351-357.

Cohen, J. D., Dunbar, K., \& McClelland, J. L. (1990). On the control of automatic processes: A parallel distributed processing account of the Stroop effect. Psychological Review, 97, 332-361.

De Jong, R. (2000). An intention-activation account of residual switch costs. In S. Monsell \& J. Driver (Eds.), Control of cognitive processes: Attention and performance XVIII (pp. 357-376). Cambridge, MA: MIT Press.

De Jong, R., Liang, C.-C., \& Lauber, E. (1994). Conditional and unconditional automaticity: A dual-process model of effects of spatial stimulus-response correspondence. Journal of Experimental Psychology: Human Perception \& Performance, 20, 731-750.

FAIRWEATHER, H. (1978). Choice reaction times in children: Error and post-error responses, and the repetition effect. Journal of Experimental Child Psychology, 26, 407-418.

Gratton, G., Coles, M. G. H., Sirevaag, E. J., Eriksen, C. W., \& Donchin, E. (1988). Pre- and poststimulus activation of response channels: A psychophysiological analysis. Journal of Experimental Psychology: Human Perception \& Performance, 14, 331-344.

Hommel, B. (1998). Automatic stimulus-response translation in dualtask performance. Journal of Experimental Psychology: Human Perception \& Performance, 24, 1368-1384.

Hübner, R., Futterer, T., \& Steinhauser, M. (2001). On attentional control as a source of residual shift costs: Evidence from twocomponent task shifts. Journal of Experimental Psychology: Learning, Memory, \& Cognition, 27, 640-653. 
James, W. (1890). The principles of psychology. New York: Henry Holt.

Kornblum, S., HasbroucQ, T., \& Osman, A. (1990). Dimensional overlap: Cognitive basis for stimulus-response compatibility-A model and taxonomy. Psychological Review, 97, 253-270.

LAMING, D. (1979). Choice reaction performance following an error. Acta Psychologica, 43, 199-224.

Lavie, N. (2005). Distracted and confused? Selective attention under load. Trends in Cognitive Sciences, 9, 75-82.

Lavie, N., Hirst, A., De Fockert, J. W., \& Viding, E. (2004). Load theory of selective attention and cognitive control. Journal of Experimental Psychology: General, 133, 339-354.

Lhermitte, F. (1983). "Utilization behaviour" and its relation to lesions of the frontal lobes. Brain, 106, 237-255.

Logan, G. D. (1988). Toward an instance theory of automatization. Psychological Review, 95, 492-527.

Lu, C.-H., \& Proctor, R. W. (1995). The influence of irrelevant location information on performance: A review of the Simon and spatial Stroop effects. Psychonomic Bulletin \& Review, 2, 174-207.

McClelland, J. L. (2001). Failures to learn and their remediation: A Hebbian account. In J. L. McClelland \& R. S. Siegler (Eds.), Mechanisms of cognitive development: Behavioral and neural perspectives (pp. 97-122). Mahwah, NJ: Erlbaum.

MeIran, N. (1996). Reconfiguration of processing mode prior to task performance. Journal of Experimental Psychology: Learning, Memory, \& Cognition, 22, 1423-1442.

MeIran, N. (2000). Modeling cognitive control in task-switching. Psychological Research, 63, 234-249.

Meiran, N., Chorev, Z., \& SAPIr, A. (2000). Component processes in task switching. Cognitive Psychology, 41, 211-253.

Meiran, N., \& Daichman, A. (2005). Advance task preparation reduces task error rate in the cuing task-switching paradigm. Memory \& $\mathrm{Cog}_{-}$ nition, 33, 1272-1288.

MonselL, S. (1996). Control of mental processes. In V. Bruce (Ed.), Unsolved mysteries of the mind: Tutorial essays in cognition (pp. 93-148). Hove, U.K.: Erlbaum.

Norman, D. A. (1981). Categorization of action slips. Psychological Review, 88, 1-15.

Norman, D. A., \& Shallice, T. (1986). Attention to action: Willed and automatic control of behaviour. In R. J. Davidson, G. E. Schwartz, \& D. Shapiro (Eds.), Consciousness and self-regulation: Advances in research and theory (Vol. 4, pp. 1-18). New York: Plenum.

Philipp, A. M., Joliceleur, P., Falkenstein, M., \& Koch, I. (2007). Response selection and response execution in task switching: Evidence from a go-signal paradigm. Journal of Experimental Psychology: Learning, Memory, \& Cognition, 33, 1062-1075.

RabBiTT, P. M. A. (1968). Three kinds of error-signalling responses in a serial choice task. Quarterly Journal of Experimental Psychology, 20, 179-188.

Reason, J. (1990). Human error. Cambridge: Cambridge University Press.

RIDDERINKHOF, K. R. (2002). Micro- and macro-adjustments of task set: Activation and suppression in conflict tasks. Psychological Research, 66, 312-323.

RidDerinkhof, K. R., Scheres, A., Oosterlaan, J., \& Sergeant, J. A. (2005). Delta plots in the study of individual differences: New tools reveal response inhibition deficits in $\mathrm{AD} / \mathrm{HD}$ that are eliminated by methylphenidate treatment. Journal of Abnormal Psychology, 114, 197-215.

RidDerinkHof, K. R., VAN DEN WiLdEnberG, W. P. M., WiJnen, J., \& BurLe, B. (2004). Response inhibition in conflict tasks is revealed in delta plots. In M. I. Posner (Ed.), Cognitive neuroscience of attention (pp. 369-377). New York: Guilford.

Rogers, R. D., \& Monsell, S. (1995). Costs of a predictable switch between simple cognitive tasks. Journal of Experimental Psychology: General, 124, 207-231.

Rubin, O., \& MeIran, N. (2005). On the origins of the task mixing cost in the cuing task-switching paradigm. Journal of Experimental Psychology: Learning, Memory, \& Cognition, 31, 1477-1491.

SChNeIDER, W., \& ShIFFrin, R. M. (1977). Controlled and automatic human information processing: I. Detection, search, and attention. Psychological Review, 84, 1-66.
SchUCH, S., \& Koch, I. (2003). The role of response selection for inhibition of task sets in task shifting. Journal of Experimental Psychology: Human Perception \& Performance, 29, 92-105.

Steinhauser, M., \& HüBNER, R. (2005). Mixing costs in task shifting reflect sequential processing stages in a multicomponent task. Memory \& Cognition, 33, 1484-1494.

Steinhauser, M., \& HÜBNER, R. (2006). Response-based strengthening in task shifting: Evidence from shift effects produced by errors. Journal of Experimental Psychology: Human Perception \& Performance, 32, 517-534.

Steinhauser, M., \& HüBner, R. (2007). Automatic activation of taskrelated representations in task shifting. Memory \& Cognition, 35, 138-155.

Steinhauser, M., Maier, M., \& HÜBner, R. (2007). Cognitive control under stress: How stress affects strategies of task-set reconfiguration. Psychological Science, 18, 540-545.

Steinhauser, M., Maier, M., \& HüBner, R. (2008). Modeling behavioral measures of error detection in choice tasks: Response monitoring versus conflict monitoring. Journal of Experimental Psychology: Human Perception \& Performance, 34, 158-176.

Vu, K.-P. L., \& Proctor, R. W. (2004). Mixing compatible and incompatible mappings: Elimination, reduction, and enhancement of spatial compatibility effects. Quarterly Journal of Experimental Psychology, 57A, 539-556.

\section{NOTES}

1. One could object that the term switch costs is somewhat misleading in this context, and should rather be replaced with repetition benefit. Indeed, the two concepts are hard to distinguish if one assumes that the strengthening of one task inevitably leads to the weakening of a competing task.

2. The number of quantiles was chosen to provide a sufficient number of trials within each quantile for calculating the means.

3. Indeed, switch benefits following errors and switch costs following correct responses even increased with an increasing base level of RT. However, this does not necessarily indicate that our error rate effects are related to a speed-accuracy trade-off. On the one hand, it is a typical phenomenon in RT distributions that RT effects increase along with base RTs (for a discussion, see Ridderinkhof, 2002; Ridderinkhof et al., 2005). On the other hand, there is no indication of a speed-accuracy trade-off in the following experiments.

4. In contrast to Experiment 1, RTs were not ranked separately for each task in Experiment 2. This method would have resulted in a rather small number of trials per cell, leading to high cell variances and a strong reduction of power. As a consequence, this would have implied that some effects did not reach significance, although the qualitative pattern of data remained the same. To test whether the type of task affected our main results at all, we reanalyzed mean performance for Task 2 on trials in which both stimuli were incongruent, using the additional variable Task 2 (magnitude, parity). No significant interaction of this variable with accuracy on trial $n-1$ and task transition was revealed.

5. Although task-repetition and task-switch trials did not differ with respect to general Task 1 performance, we obtained a smaller congruency effect for Task 1 on task-switch trials. Although this is difficult to explain, the reason for this result could also relate to the fact that more tasks are relevant on task-switch than on task-repetition trials. More specifically, the increased number of active tasks or the increased need to control task conflicts could have induced higher cognitive load on task-switch trials. Indeed, it has been shown that an increased load can enhance or reduce the processing of irrelevant information, depending on the type of the task and the type of load (see, e.g., Lavie, 2005; Lavie, Hirst, de Fockert, \& Viding, 2004).

6. Again, RTs were not ranked separately for each task, because the resulting small numbers of trials would have led to increased cell variability and reduced power. However, as in Experiment 2, adding task (magnitude, parity) as an additional variable in the analysis of mean performance did not reveal an interaction of this variable with accuracy on trial $n-1$ and task transition, in either the short- or the long-RCI condition.

(Manuscript received December 22, 2006; revision accepted for publication January 9, 2008.) 